in vivo $34: 917-921(2020)$

doi:10.21873/invivo.11858

\title{
Eribulin Suppresses New Metastases in Patients With Metastatic Breast Cancer
}

\author{
TAKAAKI FUJI, SHOKO TOKUDA, YUKO NAKAZAWA, SASAGU KUROZUMI, \\ SAYAKA OBAYASHI, REINA YAJIMA and KEN SHIRABE \\ Division of Breast and Endocrine Surgery, Department of General Surgical Science, \\ Graduate School of Medicine, Gunma University, Gunma, Japan
}

\begin{abstract}
Background/Aim: This study aimed to investigate the progression type of metastatic breast cancer $(M B C)$ in patients undergoing eribulin chemotherapy. Materials and Methods: We retrospectively investigated the cases of 66 consecutive patients with $M B C$ who underwent eribulin chemotherapy. Results: A total of 15 patients (22.7\%) received eribulin as a $3^{\text {rd }}$-line or later treatment, and 17 (25.8\%) received eribulin as a $1^{\text {st }}$-line treatment. The overall response was complete response in $0(0 \%)$, partial response in 15 (22.7\%), stable disease in 27 (40.9\%), and progressive disease in $24(36.4 \%)$ patients. By the time of data cut-off, time to treatment failure (TTF) events had been observed in 60 patients (90.9\%), among whom, 15 (25\%) had disease progression due to NM, and 45 (75\%) had disease progression due to PL. In the regimen before eribulin administration, among 49 patients, 24 (49.0\%) had disease progression due to NM. Luminal-type patients and those with triple-negative breast cancer exhibited a similar tendency, i.e., the rate of NM was lower in the patients treated with eribulin. The rate of NM was lower in the patients

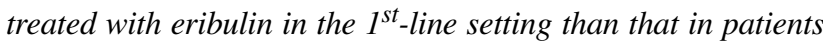
treated with eribulin as a later treatment. Conclusion: Eribulin has a potential antitumor mechanism to prevent new metastasis. Eribulin may be effective against both the epithelialmesenchymal transition (EMT) process and new metastasis.
\end{abstract}

Breast cancer (BC) is the most frequently diagnosed malignant disease among women and one of the most common causes of cancer-related death (1). Several

This article is freely accessible online.

Correspondence to: Takaaki Fujii, MD, Ph.D., FACS, Department of General Surgical Science, Graduate School of Medicine, Gunma University, 3-39-22 Showa-machi, Maebashi, Gunma 371-8511, Japan. Tel: +81 0272208224, Fax: +81 0272208230, e-mail: ftakaaki@gunma-u.ac.jp

Key Words: Eribulin, new metastasis, breast cancer. treatments are available for patients with metastatic breast cancer (MBC), but there is no cure for MBC. The overall survival (OS) of patients with MBC has improved recently, and a great need exists for treatments that further improve the OS of patients with MBC.

Eribulin is a novel microtubule target agent that is used in the treatment of $\operatorname{MBC}(2,3)$. The EMBRACE clinical trial showed a survival advantage for patients who had received between two and five previous chemotherapy regimens, including an anthracycline and a taxane, with MBC who were treated with eribulin, compared with treatment of physician's choice (TPC) (3-5). In that trial, there was a significant improvement in the OS of eribulin-treated patients compared to those treated with TPC (4). Interestingly, two different $\mathrm{MBC}$ clinical trials including the EMBRACE trial have suggested that eribulin has more pronounced effects on OS compared to progression-free survival (PFS) (3-7). One possible explanation for this clinical observation is that eribulin may suppress the incidence of new metastasis (NM) (6).

The concepts of progression due to pre-existing lesions (PLs) and progression due to new metastasis were recently proposed to differentiate the progression type of treatmentresistant cancers (6). We conducted the present study to investigate the progression type of $\mathrm{MBC}$ in patients undergoing eribulin chemotherapy.

\section{Patients and Methods}

Patients. We retrospectively investigated the cases of 66 consecutive patients with MBC who underwent eribulin chemotherapy at Gunma University Hospital between December 2011 and July 2017. Patients with incomplete clinical information and male patients were excluded. There were no exclusions based on age or menopausal status. Using the patients' medical records, we collected the characteristics of the primary tumors and metastatic tumors including the estrogen receptor (ER) and progesterone receptor $(\mathrm{PgR})$ expression status, the human epidermal growth factor receptor 2 (HER2) score, and the molecular subtypes (luminal, luminal-HER2, HER2-enriched, and triple-negative breast cancer (TN). Metastatic sites including lymph nodes and distant organs 
were also recorded and analyzed. Written consent for the use of their records and imaging in future studies was obtained from all patients, and the study was approved by our Clinical Ethics Committee (No. 1297).

Treatment. The treatment consisted of monotherapy with intravenous eribulin, which was administered at a dose of $1.4 \mathrm{mg} / \mathrm{m}^{2}$ body surface on days 1 and 8 of the subsequent cycles, every 3 weeks. This regimen was continued until the occurrence of progressive disease (PD), as assessed by the investigator using the RECIST criteria, or the appearance of unmanageable toxicity, or the patient's withdrawal from treatment. Any concomitant medication could be given at the discretion of the investigator if it was considered necessary for the patient's welfare and was not expected to interfere with the evaluation of the study treatment.

Assessments and statistical analyses. We assessed the objective response rate (ORR) by using the RECIST criteria. The ORR was defined as the proportion of all patients with a complete response $(\mathrm{CR})$ or a partial response (PR). Each $\mathrm{CR}$ and $\mathrm{PR}$ required confirmation $\geq 4$ weeks after first being reported. A pre-existing lesion (PL) was defined as PD by progression due to pre-existing lesions and does not involve metastasis. The NM status was defined as PD due to metastasis to other organs. We compared between patients treated with eribulin and patients who treated with regimen before eribulin administration. To compare two groups, we used Fisher's exact test or the $\chi^{2}$ test with Yates' correction or a one-way analysis of variance (ANOVA). Differences were considered significant when $p<0.05$.

\section{Results}

A total of 66 patients that received at least one dose of eribulin were included in the analysis. Table I summarizes the patients' characteristics. The median age was 54.0 years (range $=35-80$ years). The analysis of the molecular subtypes indicated that $43(65.2 \%)$ were luminal type, three $(4.5 \%)$ were luminal-HER2, three (4.5\%) were HER2-enriched, and $17(25.8 \%)$ were TN. The common sites of metastasis were as follows; liver $(\mathrm{n}=38,57.6 \%)$, lung $(\mathrm{n}=35,53.0 \%)$, bone $(\mathrm{n}=29,43.9 \%)$, lymph nodes $(\mathrm{n}=19,28.8 \%)$, and brain $(\mathrm{n}=13,19.7 \%)$. The median number of previous chemotherapy regimens was 1 (range $=0-6$ ).

A total of $31(47.0 \%)$ received eribulin as a $3^{\text {rd }}$-line or later treatment, and $17(25.8 \%)$ received eribulin as a $1^{\text {st }}$. line treatment. The median course of eribulin treatment was 7.5 (range $=2-21)$ courses. The overall response was CR in $0(0 \%), \mathrm{PR}$ in $15(22.7 \%), \mathrm{SD}$ in $27(40.9 \%)$, and PD in 24 (36.4\%) patients. By the time of the data cut-off, time to treatment failure (TTF) events had been observed in 60 of the 66 patients $(90.9 \%)$. Among these 60 patients, 15 patients $(25 \%)$ had disease progression due to NM, and 45 patients $(75 \%)$ had disease progression due to PL. In contrast, in the regimen before eribulin administration, among 49 patients (other than the 17 patients with $1^{\text {st }}$-lineeribulin), $24(49.0 \%)$ had disease progression due to $\mathrm{NM}$ (Figure 1).
Table I. Characteristics of patients treated with eribulin $(n=66)$.

\begin{tabular}{lc}
\hline Age median (range), (years) & $54(35-80)$ \\
Metastatic lesion, (n) & 13 \\
1 & 25 \\
2 & 28 \\
$3<$ & \\
Subtype, (n) & 43 \\
Luminal & 3 \\
Luminal-HER2 & 3 \\
HER2-enriched & 17 \\
Triple negative & \\
Metastatic sites, (n) & 38 \\
Liver & 35 \\
Lung & 29 \\
Bone & 19 \\
Lymph node & 13 \\
Brain & 3 \\
Local lesion & 14 \\
Other & \\
Priory chemotherapy, (n) & 17 \\
0 & 18 \\
1 & 31 \\
$2<$ &
\end{tabular}

In the group of 43 luminal-type patients, a total of 10 $(23.3 \%)$ received eribulin as a $3^{\text {rd }}$-line or later treatment, and $11(25.6 \%)$ received eribulin as a $1^{\text {st }}$-line treatment. The median course of eribulin treatment was 8.5 courses (range $=2-15$ courses). By the time of the data cut-off, TTF events had been observed in 37 patients $(86.0 \%)$, among whom eight patients $(21.6 \%)$ had disease progression due to $\mathrm{NM}$, and 29 patients $(78.4 \%)$ had disease progression due to PL. In contrast, in the regimen before eribulin administration, among 32 patients (other than the 11 eribulin $1^{\text {st }}$-line patients), 14 (43.8\%) had disease progression due to NM (Figure 2a).

In the group of 17 patients with $\mathrm{TN}$, three (17.6\%) received eribulin as a $3^{\text {rd }}$-line or later treatment and five $(29.4 \%)$ received eribulin as a $1^{\text {st }}$-line treatment. The median course of eribulin treatment was 5.5 courses (range $=2-21$ courses). By the time of the data cut-off, TTF events had been observed in all 17 patients. Among the 17 patients, five (29.4\%) had disease progression due to NM, and 12 patients (70.6\%) had disease progression due to PL. In the regimen before eribulin administration, among 12 patients (other than the five eribulin $1^{\text {st }}$-line patients), nine $(75.0 \%)$ had disease progression due to NM (Figure 2b).

The characteristics of the patients in the three treatment settings are summarized in Table II. In the $1^{\text {st }}$-line setting, among 13 patients, only one (7.7\%) had disease progression due to NM. In the $2^{\text {nd }}$-line setting, among 16 patients, five $(31.3 \%)$ had disease progression due to NM. In the $3^{\text {rd }}$-line or later treatment, among 31 patients, eight $(25.8 \%)$ had disease progression due to NM (Figure 3). 


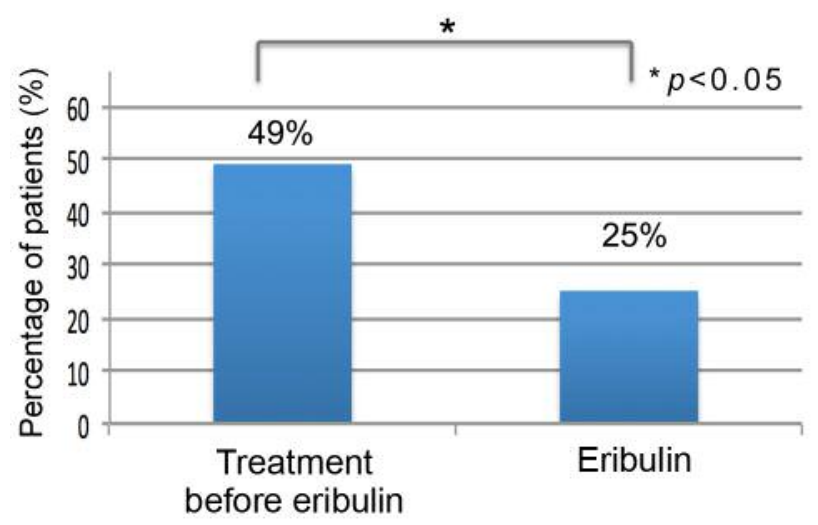

Figure 1. The rate of new metastasis (NM) in disease progression. Among 60 patients treated with eribulin, 15 patients (25\%) had disease progression due to $N M$, and 45 patients (75\%) had disease progression due to pre-existing lesion (PL). In contrast, in the regimen before eribulin administration, among 49 patients (other than the 17 patients

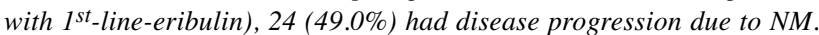
The rate of $N M$ was higher in patients treated with elibulin compared to those treated before eribulin $(p<0.05)$.

\section{Discussion}

Eribulin has provided a significant improvement in the overall survival of patients with MBC (4). Interestingly, eribulin has shown more pronounced effects on OS compared to PFS (4-7). Some research groups have reported that eribulin has additional antitumor mechanisms beyond its established antimitotic activity. One possible explanation for this clinical observation is that eribulin may suppress the incidence of new metastases (6). The key observations of the present study can be summarized as follows: 1) the rate of disease progression due to $\mathrm{NM}$ was lower in patients treated with eribulin $(25.0 \%)$ than that in patients treated before eribulin administration $(49.0 \%)$; 2) luminal-type and TN patients exhibited a similar tendency, i.e., the rate of NM was lower in the patients treated with eribulin; and 3) the rate of $\mathrm{NM}$ was lower in the patients treated with eribulin in the $1^{\text {st }}$ line setting compared to the rate in patients treated with eribulin as a later treatment. Our results suggest that eribulin has a potential antitumor mechanism to prevent new metastases.

The epithelial-mesenchymal transition (EMT) is considered a crucial process in tumor progression and metastasis (8-12). The EMT has been shown to have critical roles in both tumor invasion and metastasis. During the EMT, the gene signature and phenotype of epithelial cells change in such a way that they adopt mesenchymal characteristics that have been implicated in enhanced tumor invasion and metastasis, and a shift toward stem cell phenotypes $(3,11)$. Preclinical studies have indicated that eribulin treatment makes render residual a

\section{Luminal}
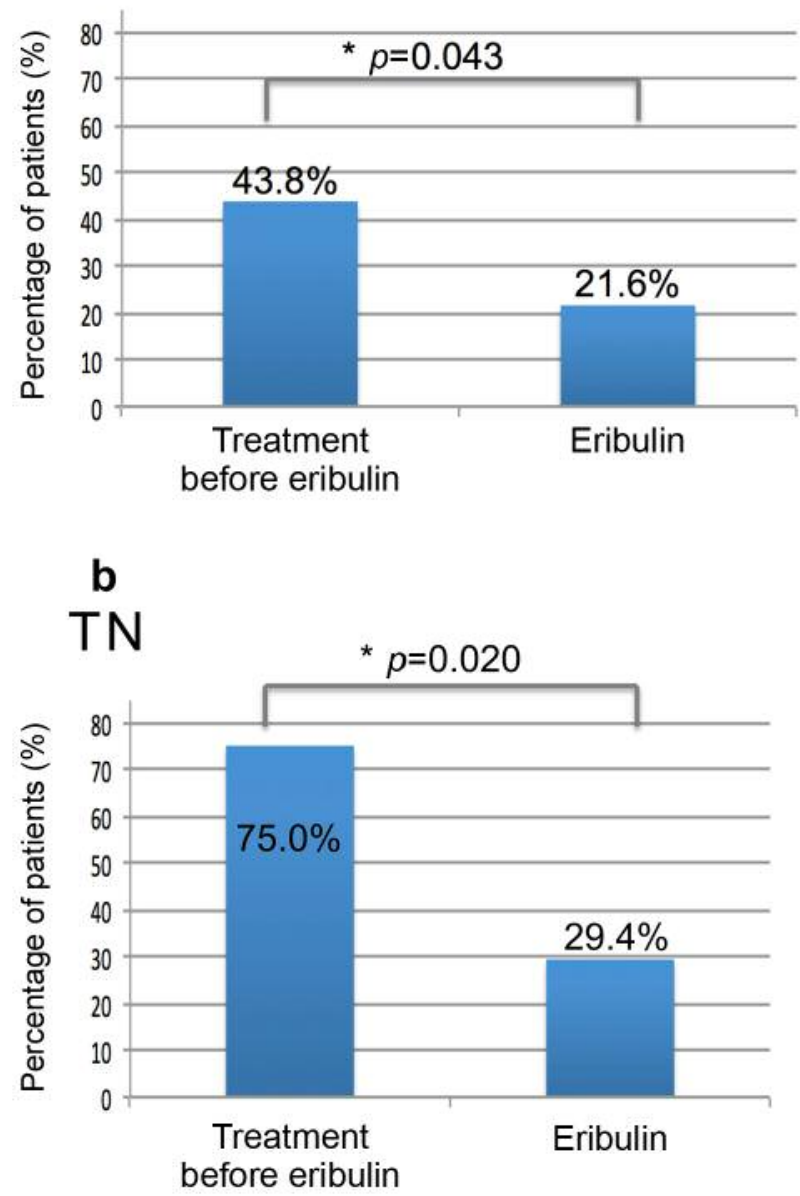

Figure 2. The rate of new metastasis (NM) in disease progression in subgroups. $a$. The rate of new metastasis (NM) in disease progression in patients with luminal-type breast cancer. Among 37 patients treated with eribulin, eight patients $(21.6 \%)$ had disease progression due to $N M$, and 29 patients (78.4\%) had disease progression due to PL. In contrast, in the regimen before eribulin administration, among 32 patients (other than the 11 eribulin 1st-line patients), 14 (43.8\%) had disease progression due to NM. $b$. The rate of new metastasis (NM) in disease progression in patients with triple negative breast cancer. Among the 17 patients with disease progression, five (29.4\%) had disease progression due to NM, and 12 patients (70.6\%) had disease progression due to PL. In the regimen before eribulin administration, among 12 patients (other than the five eribulin $1^{\text {st-line patients), nine }}$ (75.0\%) had disease progression due to NM.

tumors less likely to metastasize by triggering a shift from mesenchymal to epithelial phenotypes via a reversal of the EMT state to the mesenchymal-epithelial transition (MET) state $(10,12)$. These specific characters of eribulin on the EMT-MET balance may be associated with progression due to new metastasis (6). 
Table II. Characteristics of patients in the three treatment settings.

\begin{tabular}{lccc}
\hline & $\begin{array}{c}1^{\text {st-line }} \\
\mathrm{n}=17\end{array}$ & $\begin{array}{c}2^{\text {nd }} \\
\mathrm{n}=18\end{array}$ & $\begin{array}{c}3^{\text {rd }} \text {-line or } \\
\text { later treatment } \\
\mathrm{n}=31\end{array}$ \\
\hline $\begin{array}{l}\text { Age median (range), (years) } \\
\text { Subtype, (n) }\end{array}$ & $54.5(35-80)$ & $53(35-80)$ & $54(35-80)$ \\
Luminal & 11 & 14 & 18 \\
Luminal-HER2 & 1 & 0 & 2 \\
HER2-enriched & 0 & 1 & 2 \\
Triple negative & 5 & 3 & 9 \\
Overall response, (n) & & & \\
CR & 0 & 0 & 0 \\
PR & 5 & 5 & 4 \\
SD & 6 & 10 & 12 \\
PD & 6 & 3 & 15 \\
ORR (\%) & 29.4 & 27.7 & 12.9 \\
\hline
\end{tabular}

CR: Complete response; PR: partial response; SD: stable disease; PD: progressive disease; ORR: objective response rate.

Progression due to NM has been shown to be associated with significantly worse overall survival in patients with MBC (6). Our present findings support the hypothesis that eribulin contributes to the prevention of new metastases.

In clinical trials of patients with late-stage MBC, eribulin has shown trends towards greater OS compared to PFS (47). The authors of Study 301 and Study 305/EMBRACE reported that approximately one-third of their patients developed new metastases and over one-quarter experienced progression due to the growth of pre-existing lesions (4-7). In the present retrospective study, among the patients treated with eribulin as the $1^{\text {st }}$-line therapy, only one patient had disease progression due to NM, and in the later treatment settings, less patients had disease progression due to NM. Our results thus suggest that eribulin may be effective for preventing new metastases even in early-phase treatment. On the other hand, eribulin may remodel aspects of the tumor microenvironment (such as the EMT), which may improve its antitumor activity and may also potentially impact the effects of concomitant or subsequent anticancer therapies.

This study has several limitations. It was a retrospective analysis, and the number of cases was relatively small $(n=66)$. Additional research is needed to explore the hypothesis that eribulin prevents new metastases in metastatic breast cancer. However, few real-world experiences with eribulin treatment have been reported (13), and to the best of our knowledge this is the first report to describe an additional role of eribulin in real-world breast cancer treatment.

In conclusion, the results of our analyses demonstrated that eribulin has a potential antitumor mechanism to prevent new metastases in patients with metastatic breast cancer. Eribulin may affect the EMT-MET balance and progressive

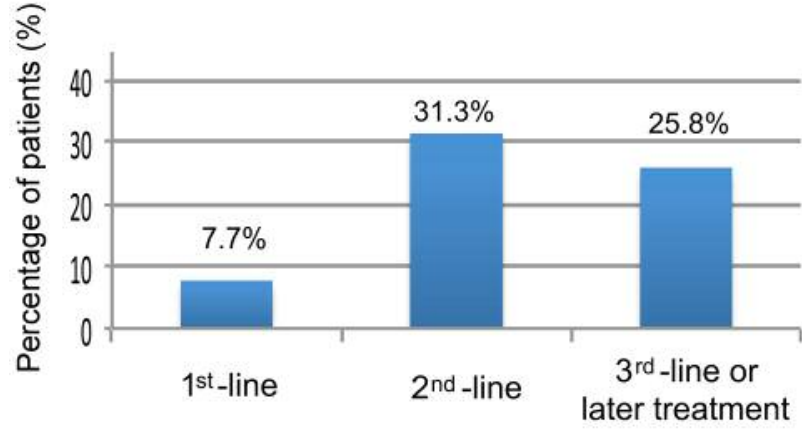

Figure 3. The rate of new metastasis (NM) in disease progression in patients in the three treatment settings. In the $1^{\text {st }}$-line setting, among 13 patients, one $(7.7 \%)$ had disease progression due to NM. In the $2^{\text {nd }}$-line setting, among 16 patients, five (31.3\%) had disease progression due to

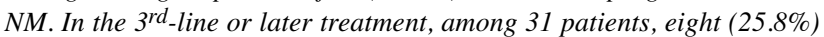
had disease progression due to NM.

disease due to new metastasis or pre-existing lesion progression. Analyses from large randomized trials are warranted to evaluate the relationship between eribulin treatment and new metastasis.

\section{Conflicts of Interest}

The Authors declare that they have no competing financial interests regarding this study.

\section{Authors' Contributions}

TF analyzed data and wrote the initial draft of the manuscript. ST, YN, SG, SO, and RY collected data and were involved in the initial study conception and design. ST, YN, SG, SO, RY, and SK were involved in drafting and revising the manuscript. All Authors have read and approved the final manuscript.

\section{Acknowledgements}

The Authors would like to thank Saitoh Y, Takata F and Kanai H for their secretarial assistance. This work was supported by Grantsin-Aid from the Japanese Ministry of Education, Culture, Sports, Science and Technology (T.F.).

\section{References}

1 Ferlay J, Soerjomataram I, Dikshit R, Eser S, Mathers C, Rebelo M, Parkin DM, Forman D and Bray F: Cancer incidence and mortality worldwide: Sources, methods and major patterns in GLOBOCAN 2012. Int J Cancer 136: E359-86, 2015. PMID: 25220842. DOI: $10.1002 /$ ijc 29210

2 Smith JA, Wilson L, Azarenko O, Zhu X, Lewis BM, Littelefield BA and Jordan MA: Eribulin binds at microtubule ends to a single 
site on tubulin to suppress dynamic instability. Biochemistry 46: 1331-1337, 2010. PMID: 20030375. DOI: 10.1021/bi901810u

3 Dybdal-Hargreaves NF, Risinger AL and Mooberry SL: Eribulin Mesylate: Mechanism of action of a unique microtubule targeting agent. Clin Cancer Res 21: 2445-2452, 2015. PMID: 25838395. DOI: 10.1158/1078-0432.CCR-14-3252

4 Cortes J, O'Shaughnessy J, Loesch D, Blum JL, Vahdat LT, Petrakova K, Chollet P, Manikas A, Diéras V, Delozier T, Vladimirov V, Cardoso F, Koh H, Bougnoux P, Dutcus CE, Seegobin S, Mir D, Meneses N, Wanders $J$ and Twelves C; EMBRACE (Eisai Metastatic Breast Cancer Study Assessing Physician's Choice Versus E7389) investigators: Eribulin monotherapy versus treatment of physician's choice in patients with metastatic breast cancer (EMBRACE): a phase 3 open-label randomised study. Lancet 377: 914-923, 2011. PMID: 21376385. DOI: 10.1016/S0140-6736(11)60070-6

5 Twelves C, Cortes J, Vahdat L, Olivo M, He Y, Kaufman PA and Awada A: Efficacy of eribulin in women with metastatic breast cancer: a pooled analysis of two phase 3 studies. Breast Cancer Res Treat 148: 553-561, 2014. PMID: 25381136. DOI: 10.1007/ s10549-014-3144-y

6 Twelves C, Cortes J, Kaufman PA, Yelle L, Awada A, Binder TA, Olivo M, Song J, O'Shaughnessy JA, Jove M and Perez EA: "New" metastases are associated with a poorer prognosis than growth of pre-existing metastases in patients with metastatic breast cancer treated with chemotherapy. Breast Cancer Res Treat 17: 150, 2015. PMID: 27391598. DOI: 10.1186/s13058015-0657-1

7 Kaufman PA, Awada A, Twelves C, Yelle L, Velikova G, Olivo MS, He Y, Dutcus CE and Cortes J: Phase III open-label randomized study of eribulin mesylate versus capecitabine in patients with localy advanced or metastatic breast cancer previously treated with an anthracycline and a taxan. J Clin Oncol 33: 594-601, 2015. PMID: 25605862. DOI: 10.1200/ JCO.2013.52.4892
8 Yang J and Weinberg RA: Epithelial-mesenchymal transition: at the crossroads of development and tumor metastasis. Dev Cell 14: 818-829, 2008. PMID: 18539112. DOI: 10.1016/ j.devcel.2008.05.009

9 Gunasinghe NP, Wells A, Thompson EW and Hugo HJ: Mesenchymal-epithelial transition (MET) as amechanism for metastatic colonization in breast cancer. Cancer Metastasis Rev 31: 469-478, 2012. PMID: 22729277. DOI: 10.1007/s10555012-9377-5

10 Yohida T, Ozawa Y, Kimura T, Sato Y, Kuznetsov G, Xu S, Uesugi M, Agoulnik S, Taylor N, Funahashi Y and Matsui J: Eribulin mesialte suppresses experimental metastasis of breast cancer cells by reversing phenolype from eptithelialmesenchymal transition (EMT) to mesenchymeal-epithelial transition (MET) states. Br J Cancer 110: 1497-1505, 2014. PMID: 24569463. DOI: 10.1038/bjc.2014.80

11 Steinestel K, Eder S, Schrader AJ and Steinestel J: Clinical significance of epithetial-mesenchymal transition. Clin Trans Med 3: 17, 2014. PMID: 25050175. DOI: 10.1186/2001-1326-3-17

12 Terashima M, Sakai K, Togashi Y, Hayashi H, Velasco MA, Tsurutani J and Nishio K: Synergistic antitumor effects of S-1 with eribulin in vitro and in vivo for triple-negative breast cancer cell lines. SpringerPlus 3: 417, 2014. PMID: 25140293. DOI: 10.1186/2193-1801-3-417

13 Watanabe J: Eribulin monotherapy improved survivals in patients with ER-positive HER2-negative metastatic breast cancer in the real world: a single institutional review. Springerplus 4: 625, 2015. PMID: 26543760. DOI: 10.1186/ s40064-015-1422-8
Received November 26, 2019

Revised December 10, 2019

Accepted December 13, 2019 